\title{
DISSEMINATED HISTOPLASMOSIS AS PRESENTING FEATURE OF ACQUIRED IMMUNODEFICIENCY SYNDROME: A CASE REPORT
}

\author{
ROKSANA YASMIN ${ }^{1}$, MUHAMMAD ABDUR RAHIM ${ }^{2}$, HASNA FAHMIMA HAQUE ${ }^{3}$, PRATIK DEWAN ${ }^{3}$, \\ JAMAL UDDIN AHMED ${ }^{2}$, AKM SHAHEEN AHMED ${ }^{4}$, MD. DELWAR HOSSAIN ${ }^{5}$, AKM MUSA ${ }^{5}$, \\ KHWAJA NAZIM UDDIN ${ }^{6}$
}

\begin{abstract}
Histoplasmosis is a mild self-limiting respiratory illness in immune-competent individuals. It disseminates in immune-suppressed states like patients with human immunodeficiency virus (HIV) infection, haematological malignancies, post transplant states and patients receiving immunesuppressive therapy. Here we report a case of disseminated histoplasmosis in a 45 year old diabetic, returning worker who presented with fever, cough, rash, oro-genital ulcer and weight loss. He was anaemic, there was crepitation on lung auscultation and hepatomegaly. Diagnostic workup revealed HIV infection with disseminated histoplasmosis. Intravenous amphotericin B was started along with other supportive therapy and for anti-retroviral therapy he was sent to a referral center. Being an uncommon condition in our perspective, we are reporting the case.
\end{abstract}

Key words: Disseminated histoplasmosis, AIDS, immunocompromized.

\section{Introduction}

Histoplasmosis is a fungal infection caused by Histoplasma capsulatum, which is a dimorphic, saprophytic, soil-based fungus endemic throughout the Ohio and Mississippi river valleys. ${ }^{1}$ Inspite of being non-endemic area, sporadic cases have been described in Bangladesh mostly in immunocompetent patients. ${ }^{2-5}$ Spores are found in contaminated soil and their growth is facilitated by excreta of birds and bats. In human, spores enter into the body through inhalation and then germinate into yeast form. In immune-competent individuals, infections are usually asymptomatic or mild flu-like illness can occur. But in immune-compromised hosts, it can manifest as disseminated infection. When disseminated, it can involve liver, spleen, bone marrow, lympho-reticular and gastro-intestinal systems. Skin involvement can occur in up to $10 \%$ cases with HIV infection. ${ }^{6}$ Diagnosis depends on clinical manifestation and demonstration of fungus in biopsy specimen or culture or by serological tests.

\section{Case Report}

A 45-year-old diabetic patient presented with intermittent fever reaching upto $101^{\circ} \mathrm{F}$, cough with scanty mucoid sputum production without haemoptysis and $6 \mathrm{~kg}$ weight loss over 1 month. He developed painful, multiple oral and genital ulcers which initially appeared as blisters and then ruptured. He had generalized rash. There was no history of joint pain, photosensitivity, drug intake or limb swelling. He had history of extra-marital unprotected sexual exposure while residing abroad 12 years back but he denied any homosexuality or anal intercourse in his lifetime. He was generally well before and there was nothing indicative of congenital immunodeficiency.

He was moderately anaemic and there was generalized maculo-papular rash involving extensor and flexor surfaces of limbs, trunk, palm and sole (Figure 1 and 2) and multiple tender round to oval ulcers involving lips, tongue, gum, palate, glans penis having regular margin, erythematous base and slough in the center. He did not have any lymphadenopathy. He had few bilateral crepitation scattered over whole

1. Assistant Registrar, Medicine, BIRDEM, Dhaka

2. Registrar, BIRDEM, Dhaka

3. Senior Medical Officer, BIRDEM, Dhaka

4. Assistant Professor, BIRDEM, Dhaka

5. Associate Professor, BIRDEM, Dhaka

6. Professor and Head, BIRDEM, Dhaka

Bangladesh J Medicine 2013; 24 : 78-81 


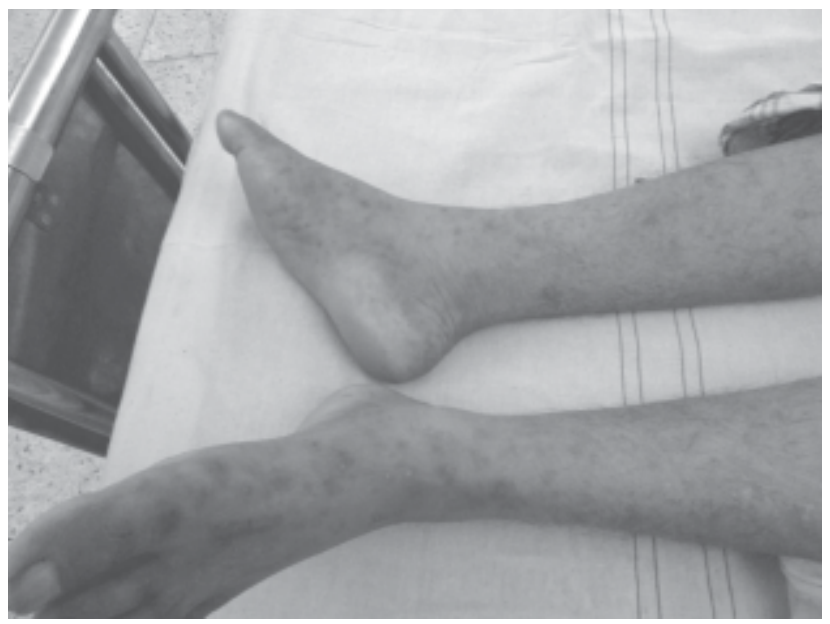

Fig.-1: Maculo-papular rash in legs, feet and sole

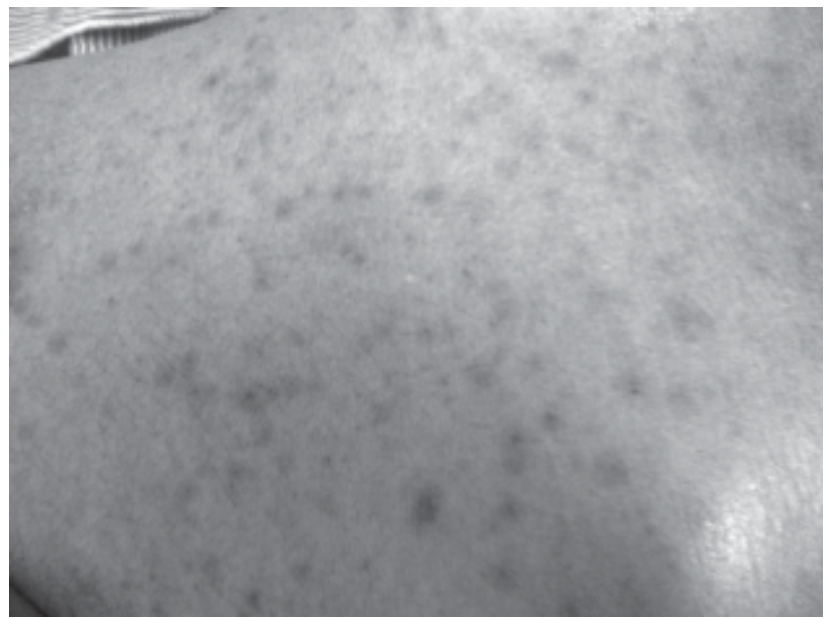

Fig.-2: Maculo-papular rash in trunk

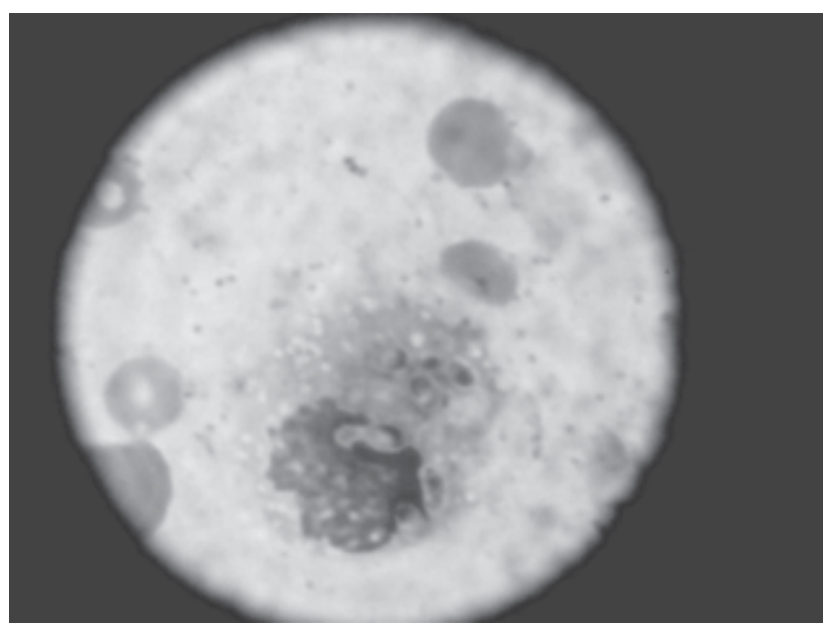

Fig.-3: Bone marrow showing intracellular Histoplasma capsulatum.

lung field. He had $6 \mathrm{~cm}$ smooth, non-tender hepatomegaly without any other organomegaly or ascites. His vital signs and other examination findings were unremarkable.
His diabetes was uncontrolled, fasting blood glucose was $9.2 \mathrm{mmol} / \mathrm{L}$ and $\mathrm{HbA} 1 \mathrm{c} 8.8 \%$. Haematological examination revealed pancytopenia; $\mathrm{Hb} 8.2 \mathrm{gm} / \mathrm{dl}$, TC of WBC $3600 / \mathrm{cmm}$ with normal differentials, platelet count $103000 / \mathrm{cmm}$, ESR $115 \mathrm{~mm}$ in $1^{\text {st }}$ hour, chest X-ray was normal, sputum Gram stain, silver stain and AFB (3 samples) were negative. Urine RME was normal. Sputum, blood and urine culture revealed no growth. Triple antigen was insignificant, MT $5 \mathrm{~mm}$ at 72 hours. ICT for kala-azar and malaria were negative. Bilirubin was $0.4 \mathrm{mg} / \mathrm{dl}$, SGPT $146 \mathrm{IU} / 1$, SGOT 537 IU/ 1, alkaline phosphatase 407 U/L, serum total protein $69 \mathrm{~g} / \mathrm{L}$, albumin $21.2 \mathrm{~g} / \mathrm{L}$, lactate dehydrogenase (LDH) $826 \mathrm{U} / \mathrm{L}$, viral markers for hepatitis $\mathrm{B}$ and $\mathrm{C}$ were negative. Abdominal ultrasonography revealed mild hepatomegaly with bright parenchyma. VDRL was non-reactive as was TPHA. Bone marrow aspirate revealed hypoplastic marrow showing proliferation of macrophages containing $H$. capsulatum (Figure 3 ), consistent with systemic histoplasmosis. Anti HIV $(1+2)$ were positive, Western blot confirmed HIV infection. His CD4 count was $04 / \mu \mathrm{L}$. HIV screen for his wife and sons were negative.

So, the patient was finally diagnosed as a case of acquired immunodeficiency syndrome (AIDS) with disseminated histoplasmosis and type 2 diabetes mellitus. He was treated with insulin, paracetamol and blood transfusion. Intravenous amphotericin B was started and for specific anti-retriviral therapy he was referred to ICDDR,B but the patient expired there on the day of admission.

\section{Discussion}

Samuel Darling reported the first case of histoplasmosis in human in December 1905 in Panama. ${ }^{7}$ Disseminated histoplasmosis (DH) was first reported in AIDS in $1982^{8}$ and was added to the United States Centers for Disease Control and Prevention (CDC) AIDS defining condition in 1987. ${ }^{9}$ Histoplasmosis is diagnosed in up to $10 \%$ of cases with AIDS in endemic areas. ${ }^{10}$

Manifestation depends on patients' immune-status and organ involvement. The majority of AIDS patients with disseminated disease have CD4 counts $<150$ cells / $\mu \mathrm{L}$, with a median CD4 count of 50 cells / $\mu \mathrm{L} .{ }^{11}$ Common features are fever, weight loss and malaise. In 50\% cases, vague respiratory symptoms are reported. ${ }^{11}$ In a review of 3 reported case series, fever, hepatomegaly, splenomegaly and generalized lymphadenopathy were the most common features (19-81\%, 19-26\%, $12.5-31 \%$ and $6-19 \%$ respectively). ${ }^{11,12}$ Skin manifestations range from 
papules to ulcers to erythema multiforme. Skin involvement is more common and more severe in Brazilian cases. ${ }^{13}$ In our case, many typical features of DH were present except lymphadenopathy and splenomegaly. CD4 count in our patient was only $4 / \mu \mathrm{L}$.

Haematological findings are mostly nonspecific. Leukopaenia, anaemia and thrombocytopaenia suggest infiltration of marrow. In our case, there was pancytopaenia and bone marrow examination revealed intracellular H. capsulatum. Elevated alkaline phosphatase may suggest hepatic infiltration and markedly elevated LDH may indicate DH in AIDS patients. ${ }^{14}$ Both enzymes were elevated in our case.

In AIDS patients, H.capsulatum can be isolated from blood and bone marrow (sensitivity 91\% and 90\% respectively). ${ }^{11}$ It can be isolated from respiratory secretions, lymph nodes, localized lessions and CSF. Culture may take upto 4 weeks, treatment should not be delayed if suspected in appropriate circumstances. Fungal stains of tissue specimens are positive in less than half of DH cases. ${ }^{15}$ Antigen can be detected in urine and serum (in 95\% and 85\% cases respectively) ${ }^{15}$, antibodies develop in 4-6 weeks but may be negative in immune-suppressive states. In countries where HIV is prevalent, identification of histoplasma infection is relatively easier. In our case, we suspected the patient as having HIV infection, but $\mathrm{DH}$ in this case was an incidental finding during work up for pancytopaenia. Chest radiograph may show multiple small, diffuse, nodular opacities and it should be noted that in up to $50 \%$ cases of $\mathrm{DH}$ chest x-ray are normal. ${ }^{16}$ CXR was normal in our patient as well.

Treatment of DH include 12 weeks induction phase with amphotericin B. Liposomal amphotericin B seems to be better than conventional amphotericin B in induction therapy (success rate $88 \%$ vs $64 \%) .{ }^{17}$ Maintenance therapy with ictraconazole is directed at preventing relapse. Without maintenance therapy relapse is common. In the era of potent antiretroviral therapy, a total of 12 month anti-fungal treatment appear to be safe in patients with sustained immunologic improvement. ${ }^{18} \mathrm{We}$ started induction phase of treatment with liposomal amphotericin B. There is no general recommendation for prophylaxis, but ictraconazole prophylaxis might be considered for those at risk for such infection.

Mortality of DH is $80 \%$ if untreated. Antifungal therapy reduces it to $25 \% .{ }^{19}$ Our patient expired 1 day after starting amphotericin $\mathrm{B}$, which might be possibly due to delay in diagnosing the case, initiationg treatment or arrhythmia as an adverse effect of systemic antifungals.

\section{Conclusion}

In an area where prevalence of HIV infection is low like Bangladesh, DH is a rare entity. Here most of the rural people keep poultry in their home and birds excreta facilitates growth of $H$. Capsulatum. If immunity is compromised, histoplasma infection can dissaminate. So, a high index of suspicion is necessary for early detection of cases.

\section{References}

1. Pastor TA, Holcomb MJ, Motaparthi K, Grekin SJ, Hsu S. Disseminated histoplasmosis mimicking secondary syphilis. Dermatology Online Journal 17 (11): 10 .

2. Islam N, Chowdhury NA. Histoplasmosis from Bangladesh: a case report. Bangladesh Med Res Counc Bull 1982;8:21-24.

3. Sarwar-E-Alam ABM, Hasan Z, Khan MAI, Zulkiful MA, Samdani ATM, Islam MT, et al. Chronic disseminated histoplasmosis in an immunocompetent man presented as bilateral adrenal masses with partial adrenocortical insufficiency- a rare condition. J Bangladesh Coll Phys Surg 2011;29:235-40.

4. Habib SK, Patwary SA, Khan MAI, Miah MT, Gupta RD, Ahsan HAMN. Primary histoplasmosis of vocal cord in an immunocompetent elderly man- a case report with literature review. J Medicine 2012;13:77-81.

5. Sadat SMA, Rita SN, Kahhar MA. Oral histoplasmosis: report of two cases. J Bangladesh Coll Phys Surg 2012;30:229-33.

6. Wheat J. Histoplasmosis in the acquired immunodeficiency syndrome. Curr Top Med Mycol 1996; 7:7-18.

7. Deepe GS. Histoplasma capsulatum.In: Mandell G, Dolin R, Bennet J, editors. Principles and practice of infectious diseases. 5 th ed. Philadelphia: Churchill Livingstone; 2000. p. 2718-32.

8. Sathapatayavongs B, Batteiger BE, Wheat J, Slama TG, Wass JL. Clinical and laboratory features of disseminated histoplasmosis during two large urban outbreaks. Medicine (Baltimore) 1983; 62:263-70.

9. Leads from the MMWR. Revision of the CDC surveillance case definition for acquired immunodeficiency syndrome. JAMA 1987; 258:1143-5, 1149, 1153-4.

10. Eidbo, J. Cutaneous manifestations of histoplasmosis in the acquired immune deficiency syndrome. Am J Surg Pathol, 1993. 17(2):p. 110-6.

11. Wheat LJ, Connolly-Stringfield PA, Baker RL, Curfman MF, Eads ME, Israel KS, et al. Disseminated histoplasmosis in the acquired immune deficiency syndrome: clinical findings, diagnosis and treatment, and review of the literature. Medicine (Baltimore) 1990; 69:361-74. 
12. Johnson PC, Khardori N, Najjar AF, Butt F, Mansell PW, Sarosi GA. Progressive disseminated histoplasmosis in patients with acquired immunodeficiency syndrome. Am J Med 1988; 85:152-8.

13. Karimi K, Wheat LJ, Connolly P, Cloud G, Hajjeh $\mathrm{R}$, Wheat $\mathrm{E}$, et al. Differences in histoplasmosis in patients with acquired immunodeficiency syndrome in the United States and Brazil. J Infect Dis 2002; 186:1655-60.

14. Corcoran GR, Al-Abdely H, Flanders CD, Geimer J, Patterson TF. Markedly elevated serum lactate dehydrogenase levels are a clue to the diagnosis of disseminated histoplasmosis in patients with AIDS. Clin Infect Dis 1997; 24:942-4.

15. Williams B, Fojtasek M, Connolly-Stringfield P, Wheat J. Diagnosis of histoplasmosis by antigen detection during an outbreak in Indianapolis, Ind. Arch Pathol Lab Med 1994; 118:1205-8.
16. Truong MT, Sabloff BS, Munden RF, Erasmus JJ. A patient with new-onset seizure and mediastinal adenopathy. Chest. 2004;126:982-985.

17. Johnson PC, Wheat LJ, Cloud GA, Goldman M, Lancaster D, Bamberger DM, et al. Safety and efficacy of liposomal amphotericin B compared with conventional amphotericin B for induction therapy of histoplasmosis in patients with AIDS. Ann Intern Med 2002; 137:105-9.

18. Goldman M, Zackin R, Fuchtenbaum CJ, Skiest DJ, Koletar SL, Hafner R et al. Safety of discontuation of maintenance therapy for disseminated histoplasmosis after immunologic response to antiretroviral therapy. Clin Infect Dis 2004; 38: 1485-9.

19. Shelburne SA 3rd, Visnegarwala F, Adams C, et al. Unusual manifestations of disseminated histoplasmosis in patients responding to antiretroviral therapy. Am J Med. 2005;118:10381041. 